\title{
COMPARISON OF THE DI- AND TRINUCLEOTIDE FREQUENCIES FROM THE GENOMES OF NINE DIFFERENT CORONAVIRUSES
}

\author{
Kurt Tobler* and Mathias Ackermann \\ Institute of Virology \\ Vet.-med. Faculty \\ University of Zurich \\ Winterthurerstrasse 266a, 8057 Zurich, Switzerland
}

\section{ABSTRACT}

As an alternative to protein alignments for the comparison of sequences, the reiterations of mono- di- and trinucleotide frequencies were used for the comparison of coronavirus sequences. The relative abundance of the di- and trinucleotide frequencies within the 3 ' part from nine coronavirus genomes were determined.

The patterns of dinucleotide frequencies and the trinucleotide frequencies showed some common features for all coronaviruses but also differences between the groups formerly defined on the base of antigenic relatedness.

The normalised dinucleotide frequencies were further used to calculate the distances between coronavirus sequences. Based on the dinucleotide frequency distances, coronaviruses can be divided into two groups which roughly reflect the taxonomic groups. In this kind of evaluation, however, IBV occupies a position different to the one that it would take based on most protein sequence comparisons. Based on similarities within coding sequences and antigenic properties IBV occupies a place outside of both groups. Based on the dinucleotide frequencies IBV gained a position in between of the TGEV-related and the MHV-clustered coronaviruses.

* Present address: BCBMB, Northwestern University, 2153, North Campus Drive, Evanston Illinois, 60208-3500.

Coronaviruses and Arteriviruses, edited by Enjuanes et al.

Plenum Press, New York, 1998 


\section{INTRODUCTION}

Evolutionary comparisons of genomes are generally based on amino acid sequence comparisons of specific proteins. Since some proteins are more conserved than others the calculated relatedness of the sequences and the implied evolutionary relationship of the organisms are strongly dependent on the sequences used for the alignment.

An alternative for the evolutionary comparison of genomes is to analyse the frequencies of di- tri- or higher order oligonucleotides. Early biochemical experiments established that the set of dinucleotide frequencies is a remarkable stable property of the DNA of an organism (Josse et al., 1961).

The frequencies of the dinucleotides were shown to be similar in related and different in non-related organisms (Burge et al., 1992; Karlin et al., 1994). These kinds of genomic compositional inhomogeneities are widely recognised. The comparison of the frequencies mirrors the correlation of the sequences. A phylogenic relationship can be constructed on the base of these values. Since the function of a genome is not confined to encoding genes, the noncoding and untranslated regions turn out to be important as well (e.g. elements used for transcription and genome replication). These parts of the genome are considered by making dinucleotide comparisons.

\section{MATERIALS AND METHODS}

The published genomic nucleotide sequences of representative coronaviruses (Porcine epidemic diarrhoea virus (PEDV), Human coronavirus 229E (229E), Transmissible gastroenteritis virus (TGEV), Porcine respiratory coronavirus (PRCV), Canine coronavirus (CCV), Bovine coronavirus (BCV), Human coronavirus OC43 (OC43), Murine hepatitis virus A59 (A59), and Infectios bronchitis virus strain Beaudette (IBV)) beginning from the start codon of the S protein to the 3' end (but excluding the poly(A) tail) were included in this study. Then the frequencies of the mononucleotides, the dinucleotides, and the trinucleotides were determined. The dinucleotide and trinucleotide frequencies were then normalized by dividing them by the frequencies of the mononucleotides contained in the dinucleotides or trinucleotide, respectively. Values of $\leq 0.78$ are considered as extreme under-representation, whereas values of $\geq 1.23$ represent extreme over-representation of a given dinucleotide frequency (Karlin et al., 1994).

The sixteen normalized dinucleotide frequencies and the sixty-four trinucleotide frequencies were used in the further calculations. The equation below describes the distances of two nucleotide sequences $g$ and $\mathrm{f}$ :

$$
\delta(f, g)=\frac{1}{N} \sum \operatorname{abs}\left[\rho_{X Y}(f)-\rho_{X Y}(g)\right]
$$

In this equation, the $\rho_{\mathrm{XY}}(\mathrm{f})$ is the normalized frequency of the dinucleotide $\mathrm{XY}$ of the sequence $\mathrm{f}$, and $\rho_{\mathrm{XY}}(\mathrm{g})$ the normalized dinucleotide frequency from the dinucleotide $\mathrm{XY}$ of the sequence g, respectively. $\mathrm{N}$ is 16 for the number of dinucleotides. Similarly, a formula for the trinucleotide could be used. There, the sum of the positive differences of the corresponding trinucleotides is divided by 64 . The resulting values describe the distances between two sequences based on the dinucleotide frequencies or trinucleotide frequencies. Lower values represent closer related sequences whereas higher values represent more distinct sequences. 
Table 1. Selected $\rho$-values of dinucleotide and trinucleotide frequencies

\begin{tabular}{lccccccccc}
\hline & PEDV & 229E & TGEV & PRCV & CCV & BCV & OC43 & A59 & IBV \\
\hline CpG & 0.60732 & 0.52635 & 0.49934 & 0.55922 & 0.50655 & 0.42774 & 0.46673 & 0.55559 & 0.51246 \\
CpA & 1.30596 & 1.31342 & 1.32916 & 1.27434 & 1.36635 & 1.16178 & 1.17393 & 1.15487 & 1.18162 \\
CpC & 0.84724 & 0.87599 & 0.87260 & 0.85933 & 0.89349 & 1.21401 & 1.16510 & 1.26549 & 1.08822 \\
GpGpC & 1.38944 & 1.37411 & 1.05993 & 1.19245 & 1.26368 & 1.14126 & 1.17165 & 1.42088 & 1.00900 \\
CpCpC & 0.66591 & 0.71401 & 0.67870 & 0.60824 & 0.82380 & 1.55631 & 1.40551 & 1.50988 & 1.08555 \\
ApCpG & 0.69475 & 0.72917 & 0.63374 & 0.77147 & 0.68903 & 0.38848 & 0.37223 & 0.41751 & 0.59202 \\
ApCpA & 1.42879 & 1.64026 & 1.66573 & 1.64538 & 1.66452 & 1.16011 & 1.14785 & 0.93200 & 1.18569 \\
ApCpC & 1.12254 & 1.23814 & 1.26219 & 1.11744 & 1.24829 & 1.45204 & 1.31410 & 1.43669 & 1.30236 \\
\hline
\end{tabular}

The theoretical values for a random sequence would be 1.0 for every $\rho$-value. Values of ${ }^{2} 0.78$ are considered as extreme underrepresentation, whereas values of ${ }^{3} 1.23$ represent extreme over-representation of a given dinucleotide frequency.

\section{RESULTS AND DISCUSSION}

The dinucleotide frequencies and some specifically chosen trinucleotide frequencies are shown in Table 1. For all sequences included in the analysis, the normalized dinucleotide frequencies revealed a constantly high value for the dinucleotide $\mathrm{UpG}$ and a low abundance for the dinucleotide $\mathrm{CpG}$. The normalised frequencies for the dinucleotide $\mathrm{CpC}$ are specifically high (around 1.2) for BCV, HCV OC43, and MHV A59 but low for the viruses PEDV, HCV 229E, TGEV, PRCV, and CCV (around 0.9). In contrast, the frequencies for the dinucleotide CpA are specifically high for the viruses PEDV, HCV 229E, TGEV, PRCV, CCV but lower for BCV, HCV OC43 and MHV A59.

Similar observations could be made by analysing the trinucleotide frequencies. The trinucleotides $\mathrm{GpGpG}, \mathrm{UpCpG}, \mathrm{CpGpG}$, and $\mathrm{CpGpU}$ are underrepresented in all coronavirus genomes included in this analysis. In contrast, an overrepresentation of the trinucleotides $\mathrm{GpUpG}, \mathrm{GpCpA}, \mathrm{CpApG}$, and $\mathrm{CpApA}, \mathrm{CpCpA}$ is common for all coronavirus genomes. The values for the trinucleotides $\mathrm{GpCpC}, \mathrm{ApCpC}$ and $\mathrm{CpCpC}$ are significantly higher in the genomes of BCV, HCV OC43, and MHV A59 than in the genomes of PEDV, HCV 229E, TGEV, PRCV, and CCV. In contrast, the trinucleotide frequencies of ApCpG and ApCpA are more abundant in the genomes of PEDV, HCV 229E, TGEV, PRCV, and CCV compared to the genomes of BCV, HCV OC43, and MHV A59.

In a consecutive analysis, the distances between the relative abundance of di- and trinucleotide frequencies of nine different coronaviruses were calculated. The distances based on the dinucleotide frequencies are presented in Table 2. Based on the distances of the dinucleotide frequencies coronaviruses can be divided into two groups which interestingly reflect the taxonomic groups. The group related to TGEV includes HCV229E, CCV, PRCV and PEDV while the other group related to MHV includes BCV and HCV OC43. In this kind of evaluation IBV occupies a position different to the one that it would take based on most protein sequence comparisons. Interestingly, based on similarities and antigenic properties IBV occupies a place outside both; but based on the dinucleotide frequencies the distances between IBV and the members of the MHV-related group are less than the distances to the members of the TGEV-clustered group and vice versa. According to the dinucleotide analyses, the ancestors of IBV might have branched later from the ancestors of the MHV-related group than the ancestors of the TGEV-clustered group.

It was shown by S. Karlin that the sequence lengths used for the analyses have only a minor effect on the results of the dinucleotide frequencies (Karlin and Burge, 1995). 
Table 2. The $\delta$-values calculated between coronavirus genomes

\begin{tabular}{lccccccccc}
\hline & PEDV & 229E & TGEV & PRCV & CCV & BCV & OC43 & A59 & IBV \\
\hline PEDV & 0 & 0.058 & 0.049 & 0.044 & 0.061 & 0.090 & 0.080 & 0.102 & 0.078 \\
229E & & 0 & 0.048 & 0.049 & 0.051 & 0.116 & 0.105 & 0.122 & 0.087 \\
TGEV & & & 0 & 0.020 & 0.026 & 0.079 & 0.072 & 0.100 & 0.061 \\
PRCV & & & & 0 & 0.037 & 0.083 & 0.078 & 0.096 & 0.065 \\
CCV & & & & & 0 & 0.089 & 0.084 & 0.103 & 0.075 \\
BCV & & & & & & 0 & 0.023 & 0.061 & 0.069 \\
OC43 & & & & & & & 0 & 0.051 & 0.059 \\
A59 & & & & & & & & 0 & 0.079 \\
IBV & & & & & & & & 0 \\
\hline
\end{tabular}

The sequence from the start codon of the $S$ gene to the poly(A) tail were used for the calculation of the $\rho$-values. Value interpretation: closely related $(\delta<0.05)$; moderately related ( $\delta$ range from 0.05 to 0.075 ); weakly related $(\delta$ range from 0.075 to 0.1$)$; distantly related $(\delta>0.1)$.

The same was observed for the coronavirus sequences. The normalized dinucleotide frequencies for the $\mathrm{N}$ gene of the viruses, for the entire genome if available, or the part of the genome from the S gene to the poly(A) tail are similar (data not shown). The longer the sequence used for calculating the frequencies, the more accurate are the values of the frequencies. The stretches of sequence used for the dinucleotide analyses of the coronaviruses are small compared to the amount of sequence taken for the comparisons of large genomes such as herpesviruses, bacteria, or eukaryotes.

\section{ACKNOWLEDGMENTS}

These studies were supported by the Swiss National Science Foundation, grant \#31-43503.95.

\section{REFERENCES}

Burge C., Campbell A.M. and Karlin S., 1992, Over- and under-representation of short oligonucleotides in DNA sequences, Proc. Natl. Acad. Sci. USA 89: 1358-1362.

Josse J., Kaiser A.D. and Kornberg A., 1961, J. Biol. Chem. 236: 864-875.

Karlin S. and Burge C., 1995, Dinucleotide relative abundance extremes: a genomic signature, Trends in Genetics 11: 283-290.

Karlin S., Ladunga I. and Blaisdell B.E., 1994, Heterogeneity of genomes: measures and values, Proc. Natl. Acad. Sci. USA 91: 12837-12841. 